
\title{
$\begin{array}{ll}\text { Research Square } & \begin{array}{l}\text { Preprints are preliminary reports that have not undergone peer review. } \\ \text { They should not be considered conclusive, used to inform clinical practice, } \\ \text { or referenced by the media as validated information. }\end{array}\end{array}$
}

\section{The C-reactive Protein to Albumin Ratio Predicts Postoperative Complication in Patients who Undergo Gastrectomy for Gastric Cancer}

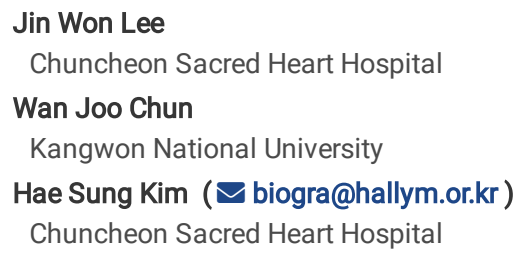

Research

Keywords: C-reactive protein to albumin ratio, gastric cancer, Postoperative complication

Posted Date: March 27th, 2020

DOI: https://doi.org/10.21203/rs.3.rs-19475/v1

License: () (i) This work is licensed under a Creative Commons Attribution 4.0 International License. Read Full License

Version of Record: A version of this preprint was published at Heliyon on June 1st, 2020. See the published version at https://doi.org/10.1016/j.heliyon.2020.e04220. 


\section{Abstract}

Background

The aim of this study was to evaluate the preoperative C-reactive protein (CRP) to albumin ratio (CAR) of patients with gastric cancer and to investigate the factors correlated with perioperative complications.

Methods

From March 2016 to December 2019, 128 patients who underwent curative gastrectomy for gastric cancer were enrolled in a retrospective study. The preoperative cutoff value of the CAR for predicting postoperative complications was 0.265 on receiver operating characteristic (ROC) curve analysis. Clinical characteristics were compared between patients with complications (Clavien-Dindo grade $\geq 2, n=20$ ) and without complications (Clavien-Dindo grade $<2, n=$ 108).

Results

On univariate and multivariate analyses, estimated blood loss $(E B L)$ during the operation $(H R 1.003, p=0.039)$ and $C A R(H R 2.832, p=0.045)$ were independent predictors of postoperative complications.

Conclusions

A high CAR was significantly associated with postoperative complications in patients with gastric cancer.

\section{Background}

Despite decreasing global incidence, gastric cancer is the fourth most common malignancy in Korea. Surgical resection with radical lymphadenectomy is the only way for cure for gastric cancer [1]. However, it has been reported that up to $46 \%$ of these patients suffer from postoperative complications (PCs) [2, 3]. Perioperative inflammation and nutritional status have been associated with postoperative complications. Although the incidence of PCs after gastrectomy has been decreasing, serious complications including reoperation, increased hospital stay and economic costs still exist. As a result, prevention of PCs is important to support individual health and economic issues.

Several studies have evaluated risk factors associated with PCs in patients undergoing gastrectomy. Among them, inflammatory markers including perioperative C-reactive protein (CRP), modified Glasgow prognostic score (mGPS) and nutritional factors have been identified as risk factors for PCs [4-10]. As a combination of these two aspects, the C-reactive protein to albumin ratio (CAR) has been discussed as a predictor for PCs in patients with several malignant diseases. However, most studies have focused on the oncologic prognostic value of CAR in patients with gastric cancer [11, 12], while only a few studies have evaluated its predictive value for PCs [13].

This study was aimed to evaluate the predictive value of the CAR for PCs in gastric cancer patients.

\section{Methods \\ Patients}

A total of 128 patients who underwent curative radical gastrectomy at Chuncheon Sacred Heart Hospital between March 2016 and December 2019 were included in this study. Demographic, preoperative, postoperative, and pathological data were collected from medical records. A complete evaluation including physical examinations, blood tests, chest X-rays, endoscopy, abdominal computed tomography scans and positron emission scanning. Pathological staging was performed using the American Joint Committee on Cancer (AJCC), seventh edition [14].

Only patients undergoing surgery with curative intent were included in this study. Patients undergoing non-curative resection, 30-day postoperative mortalities, and a history of other organ malignancies were excluded. For each patient, the following parameters were recorded: age, sex, performance status according to the Eastern Cooperative Oncology Group (ECOG) scale, body mass index (BMI), comorbidities (hypertension, DM, cardiovascular disease), tumor markers (CEA, CA 19 - 9), preoperative CEA, albumin levels, CAR, mGPS, resection extent (total gastrectomy, subtotal gastrectomy and others), and maximum tumor size (cm).

Postoperative complications were defined as complications that occurred within 30 days of the primary surgery [15]. The patients were assigned into two groups, based on the presence $(C D \geq 2, n=20)$ or absence $(C D<2, n=108)$ of postoperative complications. Patients with Clavien-Dindo (CD) grade 2 or higher complications were included in the complication group [15]. Clinicopathological characteristics were compared between the two groups.

Approval for this study was obtained from our Institutional Review Board.

\section{Cut off value of CAR}

The preoperative cutoff value of the CAR for predicting postoperative complications was 0.265 on receiver operating characteristic (ROC) curve analysis. Statistical analysis 
Continuous data were expressed as means \pm standard deviations. The independent factors significantly related to PCs were assessed using the Chi square and student t-test; the logistic regression model was performed to assess risk factors under multivariate analysis. All tests were two-sided, and statistical significance was predefined at $\mathrm{P}<0.05$. All statistical analyses were performed using SPSS software (version 12.0; SPSS, Chicago, IL, USA).

\section{Results}

\section{Patients}

The baseline characteristics of the 128 patients are shown in Table 1. The mean age was 66.8 years, and $71.1 \%(n=91)$ were male. The majority of patients had a performance status of 0 or 1 . Hypertension, diabetes mellitus (DM), and cardiovascular disease were present in 48 (37.5\%), $33(25.8 \%)$ and 16 (12.5\%) patients, respectively. At the time of surgery, the majority of patients underwent a subtotal gastrectomy (75.8\%) or total gastrectomy (21.9\%), with the remaining $2.4 \%$ undergoing other operations such as proximal gastrectomy or a Whipple's procedure. Most of the patients had a mGPS of 0 or 1 . With regard to the CAR, the number of patients with a CAR of 0.265 or less was $74(57.8 \%)$. The mean tumor size was $3.68 \mathrm{~cm}$. Based on the seventh edition of the AJCC staging system, patients with stage I tumors were the most common $(n=74,57.8 \%)$ and the remaining patients had either stage II $(n=20,15.6 \%)$ or stage III $(n=34,26.6 \%)$ tumors. Twenty of 128 patients had PCs with CD grade II or more. The overall complication rate was $15.6 \%$ and pneumonia was the most common PC $(n=6,30 \%)$. 
Table 1

Patients' clinicopathological characteristics

\begin{tabular}{|c|c|}
\hline Number & 128 \\
\hline Age (years) & $66.8 \pm 11.69$ \\
\hline $\begin{array}{l}\leq 60 \\
>60\end{array}$ & $\begin{array}{l}39(30.5) \\
(89(69.5)\end{array}$ \\
\hline $\begin{array}{l}\text { Sex } \\
\text { Male } \\
\text { Female }\end{array}$ & $\begin{array}{l}91(71.1) \\
37(28.9)\end{array}$ \\
\hline $\mathrm{BMI}\left(\mathrm{kg} / \mathrm{m}^{2}\right)$ & $24.12 \pm 3.86$ \\
\hline $\begin{array}{l}\leq 25 \mathrm{~kg} / \mathrm{m}^{2} \\
>25 \mathrm{~kg} / \mathrm{m}^{2}\end{array}$ & $\begin{array}{l}77(60.2) \\
51(39.8\end{array}$ \\
\hline $\begin{array}{l}\text { ECOG PS } \\
0 \\
1 \\
2\end{array}$ & $\begin{array}{l}81(63.3) \\
42(32.8) \\
5(3.9)\end{array}$ \\
\hline $\begin{array}{l}\text { Hypertension } \\
\text { No } \\
\text { Yes }\end{array}$ & $\begin{array}{l}80(62.5) \\
48(37.5)\end{array}$ \\
\hline $\begin{array}{l}\text { DM } \\
\text { No } \\
\text { Yes }\end{array}$ & $\begin{array}{l}95(74.2) \\
33(25.8)\end{array}$ \\
\hline $\begin{array}{l}\text { Cardiovascular disease } \\
\text { No } \\
\text { Yes }\end{array}$ & $\begin{array}{l}112(87.5) \\
16(12.5)\end{array}$ \\
\hline CEA(ng/mL) & $3.94 \pm 5.54$ \\
\hline CA $19-9(\mathrm{U} / \mathrm{mL})$ & $56.86 \pm 334.56$ \\
\hline Preoperative CRP(mg/dL) & $7.9 \pm 21.75$ \\
\hline Preoperative Albumin(g/dL) & $4.07 \pm 0.51$ \\
\hline CAR & $2.41 \pm 7.97$ \\
\hline $\begin{array}{l}\leq 0.265 \\
>0.265\end{array}$ & $\begin{array}{l}74(57.8) \\
54(42.2)\end{array}$ \\
\hline $\begin{array}{l}\text { mGPS } \\
0 \\
1 \\
2\end{array}$ & $\begin{array}{l}80(62.5) \\
38(29.7) \\
10(7.8)\end{array}$ \\
\hline Operation time(min) & $167.26 \pm 52.95$ \\
\hline $\mathrm{EBL}(\mathrm{mL})$ & $113.28 \pm 129.35$ \\
\hline $\begin{array}{l}\text { Operation Approach } \\
\text { Open } \\
\text { Laparoscopic assisted } \\
\text { Totally laparosopic } \\
\text { Robotic }\end{array}$ & $\begin{array}{l}54(42.2) \\
2(1.6) \\
68(53.1) \\
4(3.1)\end{array}$ \\
\hline $\begin{array}{l}\text { Extent of Resection } \\
\text { TG } \\
\text { STG } \\
\text { PG } \\
\text { Whipple's procedure }\end{array}$ & $\begin{array}{l}28(21.9) \\
97(75.8) \\
2(1.6) \\
1(0.8)\end{array}$ \\
\hline $\begin{array}{l}\text { LN dissection } \\
\text { D1+ } \\
\text { D2 }\end{array}$ & $\begin{array}{l}85(66.4) \\
43(33.6)\end{array}$ \\
\hline $\begin{array}{l}\text { Type of Reconstruction } \\
\text { Billroth I } \\
\text { Billroth II } \\
\text { Roux-en-Y } \\
\text { Double Tract }\end{array}$ & $\begin{array}{l}3(2.4) \\
95(74.2) \\
28(21.9) \\
2(1.55)\end{array}$ \\
\hline
\end{tabular}

Data are $\mathrm{n}(\%)$ or means \pm standard devation

BMI body mass index, ECOG PS esteran cooperative oncology group performance status, DM diabetes mellitus, CEA carcinoembryonic antigen, CA 19 - 9 carbohydrate antigen $19-9$, CAR C-reactive protein to albumin ratio, mGPS modified Glasgow prognostic score EBL estimated blood loss, TG total gastrectomy, STG subtotal gastrectomy, PG proximal gastectomy LN lymph node, AJCC American joint committee on cancer, CDC Clavian-Dindo classification, A-loop afferent loop 


\begin{tabular}{|c|c|}
\hline Number & 128 \\
\hline Tumor size(cm) & $3.68 \pm 3.05$ \\
\hline Harvested LN & $29.81 \pm 16.45$ \\
\hline Metastatic LN & $2.92 \pm 8.69$ \\
\hline $\begin{array}{l}\text { AJCC 7th Stage } \\
\text { III }\end{array}$ & $\begin{array}{l}74(57.8) \\
20(15.6) \\
34(26.6)\end{array}$ \\
\hline Length of hospital stay & $16.48 \pm 8.3$ \\
\hline Length of postoperative hospital stay & $11.71 \pm 7.13$ \\
\hline $\begin{array}{l}\text { Complication } \\
<\text { CDC grade II } \\
\geq \text { CDC grade II }\end{array}$ & $\begin{array}{l}108(84.4) \\
20(15.6)\end{array}$ \\
\hline $\begin{array}{l}\text { Grade of Complication } \\
\text { No complication } \\
\text { CDC grade I } \\
\text { CDC grade II } \\
\text { CDC grade IIIA } \\
\text { CDC grade IIIB }\end{array}$ & $\begin{array}{l}106(82.8) \\
2(1.6) \\
15(11.7) \\
1(0.8) \\
4(3.1)\end{array}$ \\
\hline $\begin{array}{l}\text { Name of Complication } \\
\text { Pneumonia } \\
\text { Bleeding } \\
\text { Intestinal obstruction } \\
\text { Pancreatic fistula } \\
\text { Intraabdominal abscess } \\
\text { Wound infection } \\
\text { A-loop syndrome } \\
\text { Gastric stasis } \\
\text { Cerebral infarction } \\
\text { Delirium }\end{array}$ & $\begin{array}{l}6(4.7) \\
1(0.8) \\
1(0.8) \\
1(0.8) \\
3(2.3) \\
3(2.3) \\
1(0.8) \\
3(2.3) \\
2(1.6) \\
1(0.8)\end{array}$ \\
\hline \multicolumn{2}{|c|}{ Data are $\mathrm{n}(\%)$ or means \pm standard devation } \\
\hline \multicolumn{2}{|c|}{$\begin{array}{l}\text { BMI body mass index, ECOG PS esteran cooperative oncology group performance status, DM diabetes mellitus, CEA carcinoembryonic antigen, CA } 19 \text { - } 9 \\
\text { carbohydrate antigen } 19-9 \text {, CAR C-reactive protein to albumin ratio, mGPS modified Glasgow prognostic score EBL estimated blood loss, TG total } \\
\text { gastrectomy, STG subtotal gastrectomy, PG proximal gastectomy LN lymph node, AJCC American joint committee on cancer, CDC Clavian-Dindo } \\
\text { classification, A-loop afferent loop }\end{array}$} \\
\hline
\end{tabular}

\section{Postoperative complications}

Baseline demographic and perioperative results associated with PCs were selected for analysis. Univariate analysis using Chi-square and student t-test identified EBL $(p=0.014)$, preoperative albumin $(p=0.028)$ and CAR $(p=0.029)$ as being significantly associated with PCs (Table 2$)$. Also, the length of the postoperative hospital stay was longer for the complication group $(10.21 \pm 3.83)$ than for the uncomplicated group $(19.80 \pm 13.24)(p=0.000)$. According to multivariate analysis using a logistic regression model, EBL and CAR were independently associated with PCs (Table 3). 
Table 2

Comparison of clinical characteristics between patients with and without postoperative complications

\begin{tabular}{|c|c|c|c|}
\hline & $\begin{array}{l}\text { Clavien-Dindo grade }<2 \\
(n=108)\end{array}$ & $\begin{array}{l}\text { Clavien-Dindo grade } \geq 2 \\
(n=20)\end{array}$ & $p$ value \\
\hline $\begin{array}{l}\text { Age } \\
\leq 60 \\
>60\end{array}$ & $\begin{array}{l}34(87.2) \\
74(83.1)\end{array}$ & $\begin{array}{l}5(12.8) \\
15(16.9)\end{array}$ & 0.611 \\
\hline $\begin{array}{l}\text { Sex } \\
\text { Male } \\
\text { Female }\end{array}$ & $\begin{array}{l}76(83.5) \\
32(86.5)\end{array}$ & $\begin{array}{l}15(16.5) \\
5(13.5)\end{array}$ & 0.792 \\
\hline $\operatorname{BMI}\left(\mathrm{kg} / \mathrm{m}^{2}\right)$ & $24.38 \pm 3.91$ & $22.70 \pm 3.30$ & 0.073 \\
\hline $\begin{array}{l}\mathrm{BMI}\left(\mathrm{kg} / \mathrm{m}^{2}\right) \\
\leq 25 \\
>25\end{array}$ & $\begin{array}{l}62(80.5) \\
46(90.2)\end{array}$ & $\begin{array}{l}15(19.5) \\
5(9.8)\end{array}$ & 0.213 \\
\hline $\begin{array}{l}\text { ECOG PS } \\
0 \\
1 \\
2\end{array}$ & $\begin{array}{l}70(86.4) \\
35(83.3) \\
2(60.0)\end{array}$ & $\begin{array}{l}11(13.6) \\
7(16.7) \\
2(40.0)\end{array}$ & 0.272 \\
\hline $\begin{array}{l}\text { Hypertension } \\
\text { No } \\
\text { Yes }\end{array}$ & $\begin{array}{l}67(83.8) \\
41(85.4)\end{array}$ & $\begin{array}{l}13(16 .) \\
7(14.6)\end{array}$ & 1.000 \\
\hline $\begin{array}{l}\text { DM } \\
\text { No } \\
\text { Yes }\end{array}$ & $\begin{array}{l}81(85.3) \\
27(81.8)\end{array}$ & $\begin{array}{l}14(14.7) \\
6(18.2)\end{array}$ & 0.781 \\
\hline $\begin{array}{l}\text { Cardiovascular disease } \\
\text { No } \\
\text { Yes }\end{array}$ & $\begin{array}{l}95(84.8) \\
13(81.3)\end{array}$ & $\begin{array}{l}17(15.2) \\
3(18.8)\end{array}$ & 1.000 \\
\hline $\mathrm{CEA}(\mathrm{ng} / \mathrm{mL})$ & $4.01 \pm 5.90$ & $3.6 \pm 2.94$ & 0.782 \\
\hline CA $19-9(\mathrm{U} / \mathrm{mL})$ & $52.68 \pm 352.35$ & $81.6 \pm 205.03$ & 0.750 \\
\hline CRP(mg/dL) & $5.12 \pm 8.86$ & $8.42 \pm 23.36$ & 0.536 \\
\hline Albumin(g/dL) & $4.12 \pm 0.52$ & $3.84 \pm 0.41$ & 0.028 \\
\hline $\begin{array}{l}\text { CAR } \\
\leq 0.265 \\
>0.265\end{array}$ & $\begin{array}{l}67(90.5) \\
41(75.9)\end{array}$ & $\begin{array}{l}7(9.5) \\
13(24.1)\end{array}$ & 0.029 \\
\hline $\begin{array}{l}\text { mGPS } \\
0 \\
1 \\
2\end{array}$ & $\begin{array}{l}71(88.8) \\
29(76.3) \\
8(80)\end{array}$ & $\begin{array}{l}9(11.3) \\
9(23.7) \\
2(20)\end{array}$ & 0.186 \\
\hline Operation time(min) & $165 \pm 51.35$ & $179 \pm 60.89$ & 0.262 \\
\hline $\mathrm{EBL}(\mathrm{mL})$ & $101.29 \pm 118.11$ & $178.0 \pm 167.5$ & 0.014 \\
\hline $\begin{array}{l}\text { Approach } \\
\text { Open } \\
\text { Lap Assisted } \\
\text { Totall Lap } \\
\text { Robotic }\end{array}$ & $\begin{array}{l}43(79.6) \\
2(100) \\
60(88.2) \\
3(75.0)\end{array}$ & $\begin{array}{l}11(20.4) \\
0(0) \\
8(11.8) \\
1(25.0)\end{array}$ & 0.529 \\
\hline $\begin{array}{l}\text { Extent of Resection } \\
\text { TG } \\
\text { STG } \\
\text { PG } \\
\text { Whipple }\end{array}$ & $\begin{array}{l}21(75.0) \\
84(86.6) \\
2(100) \\
1(100)\end{array}$ & $\begin{array}{l}7(25.0) \\
13(13.4) \\
0(0) \\
0(0)\end{array}$ & 0.350 \\
\hline $\begin{array}{l}\text { LN dissection } \\
\text { D1+ } \\
\text { D2 }\end{array}$ & $\begin{array}{l}72(84.7) \\
36(83.7)\end{array}$ & $\begin{array}{l}13(15.3) \\
7(16.3)\end{array}$ & 1.000 \\
\hline $\begin{array}{l}\text { Type of Reconstruction } \\
\text { Billloth I } \\
\text { Billroth II } \\
\text { Roux-en-Y } \\
\text { Double Tract }\end{array}$ & $\begin{array}{l}3(100) \\
82(86.3) \\
21(75.0) \\
2(100)\end{array}$ & $\begin{array}{l}0(0) \\
13(13.7) \\
7(25.0) \\
0(0)\end{array}$ & 0.379 \\
\hline
\end{tabular}

Data are $\mathrm{n}(\%)$ or means \pm standard deviation

BMI body mass index, ECOG PS esteran cooperative oncology group performance status, DM diabetes mellitus, CEA carcinoembryonic antigen, CA 19 - 9 carbohydrate antigen $19-9$, CAR C-reactive protein to albumin ratio, mGPS modified Glasgow prognostic score, EBL estimated blood loss, TG total gastrectomy, STG subtotal gastrectomy, PG proximal gastectomy LN lymph node, AJCC American Joint Committee on Cancer 


\begin{tabular}{|c|c|c|c|}
\hline & $\begin{array}{l}\text { Clavien-Dindo grade }<2 \\
(n=108)\end{array}$ & $\begin{array}{l}\text { Clavien-Dindo grade } \geq 2 \\
(n=20)\end{array}$ & $\mathrm{p}$ value \\
\hline Tumor size(cm) & $3.67 \pm 3.16$ & $3.72 \pm 2.50$ & 0.945 \\
\hline Harvested LN & $29.85 \pm 17.06$ & $29.60 \pm 13.09$ & 0.950 \\
\hline Metatatic LN & $3.25 \pm 9.39$ & $1.15 \pm 1.87$ & 0.323 \\
\hline $\begin{array}{l}\text { AJCC 7th Stage } \\
\text { II } \\
\text { III }\end{array}$ & $\begin{array}{l}65(87.8) \\
14(70.0) \\
29(85.3)\end{array}$ & $\begin{array}{l}9(12.2) \\
6(30.0) \\
5(14.7)\end{array}$ & 0.152 \\
\hline Length of hospital stay & $14.69 \pm 4.97$ & $26.15 \pm 14.33$ & 0.000 \\
\hline Postoperative hospital stay & $10.21 \pm 3.83$ & $19.80 \pm 13.24$ & 0.000 \\
\hline \multicolumn{4}{|c|}{ Data are $\mathrm{n}(\%)$ or means \pm standard deviation } \\
\hline \multicolumn{4}{|c|}{$\begin{array}{l}\text { BMI body mass index, ECOG PS esteran cooperative oncology group performance status, DM diabetes mellitus, CEA carcinoembryonic antigen, CA } 19 \text { - } \\
\text { carbohydrate antigen } 19-9 \text {, CAR C-reactive protein to albumin ratio, mGPS modified Glasgow prognostic score, EBL estimated blood loss, TG total } \\
\text { gastrectomy, STG subtotal gastrectomy, PG proximal gastectomy LN lymph node, AJCC American Joint Committee on Cancer }\end{array}$} \\
\hline
\end{tabular}

Table 3

Multivariate analysis for PCs

\begin{tabular}{|lccc|}
\hline & HR & $95 \% \mathrm{Cl}$ & P value \\
\hline $\begin{array}{l}\text { CAR } \\
\leq .265 \\
>.265\end{array}$ & 1 & $1.023-7.841$ & 0.045 \\
\hline $\mathrm{EBL}(\mathrm{mL})$ & 2.832 & & 0.039 \\
\hline HR hazard ratio, Cl confidence interval, CAR C-reactive protein to albumin ratio, EBL estimated blood loss \\
\hline
\end{tabular}

\section{Discussion}

Although radical gastrectomy is the best chance for a cure in patients with gastric cancer, PCs remain clinically relevant. Among them, infectious complications are the most common problem associated with postoperative morbidity and mortality [16]. Therefore, prediction or early detection of these complications through clinical research helps to lower the mortality and morbidity rate of gastrectomy to treat gastric cancer.

Some studies have demonstrated that inflammation or nutrition-based scores including perioperative CRP, mGPS, prognostic nutritional index (PNI) and CAR are associated with PCs after various types of surgeries $[4,5,7,8,11,13]$. Abnormal elevation of preoperative CRP reflects compromised cell-mediated immunity [17] and patients with a high preoperative CRP may be more prone to infectious complications after surgery. Also, hypoalbuminemia is a well-known factor associated with PCs due to decreased tissue healing and impaired immune response $[8,18]$. Thus, the CAR can reflect both inflammatory and immunenutritional status.

One of the most common diagnostic indicators of malnutrition is serum albumin level. Some authors insisted that a serum albumin level below $3.5 \mathrm{~g} / \mathrm{dL}$ is an independent risk factor of PCs after abdominal surgery [19]. However, the half-life of albumin is relatively long and non-nutritive conditions such as water and disease can influence the albumin level in addition to nutritional factors. Although malnutrition is associated with PCs, the albumin level cannot solely predict PCs. In our series, preoperative albumin level was significantly associated with PCs $(p=0.028)$ on univariate analysis, but multivariate analysis did not show a statistically significant predictive value of PCs after gastrectomy.

The CAR is a simple and easy marker to predict PCs after many types of surgery. Our results provided information regarding PCs in patients with a high CAR. Based on our results, patients with elevated CAR may require close observation and more intensive care after gastrectomy. These patients may also benefit from anti-inflammatory therapy or nutritional support [20,21]. Moreover, some studies have demonstrated that PCs after gastrectomy for gastric cancer are associated with long term prognosis predictors such as disease-free or overall survival rates [11]. Plus, some studies have used inflammatory mediators such as vasoactive amines and cytokines to demonstrate that inflammation is associated with tumorigenesis and metastasis [11]. Therefore, prevention of PCs based on preoperative CAR is a very important positive predictor of success.

It is possible to reverse inflammation and poor nutritional status, both of which affect PCs and hospital stay duration. The incidence of PCs can be reduced using nutritional support and anti-inflammatory therapy before and after gastrectomy for gastric cancer.

The present study has several limitations that should be considered. First, it was a retrospective, single-center study. Additionally, the optimal cutoff value for the preoperative CAR is unknown. Therefore, further large-scale and prospective multicenter studies are needed.

\section{Conclusion}

In conclusion, preoperative CAR appears to be a predictor of PCs in patients undergoing surgical treatment of gastric cancer. 


\section{Abbreviations}

CRP

C-reactive protein

CAR

C-reactive protein to albumin ratio

ROC

Receiver operating characteristics

$\mathrm{EBL}$

estimated blood loss

PCs

postoperative complications

mGPS

modified Glasgow prognostic score

AJCC

American Joint Committee on Cancer

ECOG

Eastern Cooperative Oncology Group

BMI

body mass index

DM

diabetes mellitus

CEA

carcinoembryogenic antigen

CA $19-9$

carbohydrate antigen 19 - 9

$\mathrm{CD}$

Clavien-Dindo

PNI

prognostic nutritional index

\section{Declarations}

\section{Acknowledgements}

Not applicable.

\section{Funding}

There was no funding for this study

\section{Availability of data and materials}

The material supporting the conclusion of this study has been included in the manuscript.

\section{Competing of Interests}

The authors declare that they have no competing interests

\section{Authors' contributions}

JWL carried out the conception and design, acquisition of data, analysis of data, and drafting the manuscript. WJC carried out the acquisition of data, interpretation of data, drafting the manuscript and revising it. He also participated in the design of the study and performed the statistical analysis. HSK carried out the conception and design, analysis and interpretation of data, and revising the manuscript. All authors read and approved the final manuscript.

\section{Ethics approval and consent to participate}

The institutional review board of Chuncheon Sacred Heart Hospital approved this study.

\section{Consent for publication}

Written informed consent was obtained from the patient himself.

\section{References}


1. Lee JW, Ali B, Yoo HM, Park CH, Song KY. Conditional survival analysis in Korean patients with gastric cancer undergoing curative gastrectomy. BMC Cancer. 2015;15:1005.

2. Postoperative morbidity and mortality after D1 and D2 resections for gastric cancer: preliminary results of the MRC randomised controlled surgical ... PubMed - NCBI. https://www.ncbi.nlm.nih.gov/pubmed/?

term=Postoperative+morbidity+and+mortality+after+D1+and+D2+resections+for+gastric+cancer\%3A+preliminary+results+of+the+MRC+randomised+con Accessed 6 Feb 2020.

3. Bonenkamp JJ, Songun I, Hermans J, Sasako M, Welvaart K, Plukker JT, et al. Randomised comparison of morbidity after D1 and D2 dissection for gastric cancer in 996 Dutch patients. Lancet. 1995;345:745-8.

4. Kassir R, Blanc P, Bruna Tibalbo LM, Breton C, Lointier P. C-Reactive protein and procalcitonin for the early detection of postoperative complications after sleeve gastrectomy: preliminary study in 97 patients. Surg Endosc. 2015;29:1439-44.

5. Shishido Y, Fujitani K, Yamamoto K, Hirao M, Tsujinaka T, Sekimoto M. C-reactive protein on postoperative day 3 as a predictor of infectious complications following gastric cancer resection. Gastric Cancer. 2016;19:293-301.

6. Selby J, Prabhudesai A. Can C-reactive protein predict the severity of a post-operative complication after elective resection of colorectal cancer? Int J Colorectal Dis. 2014;29:1211-5.

7. McSorley ST, Ramanathan ML, Horgan PG, McMillan DC. Postoperative C-reactive protein measurement predicts the severity of complications following surgery for colorectal cancer. Int J Colorectal Dis. 2015;30:913-7.

8. Preoperative systemic inflammation predicts postoperative infectious complications in patients undergoing curative resection for colorectal cancer. PubMed - NCBI. https://www.ncbi.nlm.nih.gov/pubmed/?

term=Preoperative+systemic+inflammation+predicts+postoperative+infectious+complications+in+patients+undergoing+curative+resection+for+colorecta Accessed 6 Feb 2020.

9. Prognostic Nutritional Index Predicts Severe Complications, Recurrence, and Poor Prognosis in Patients With Colorectal Cancer Undergoing Primary Tu... PubMed - NCBI. https://www.ncbi.nlm.nih.gov/pubmed/?

term=Prognostic+nutritional+index+predicts+severe+complications\%2C+recurrence\%2C+and+poor+prognosis+in+patients+with+colorectal+cancer+unde Accessed 6 Feb 2020.

10. Ishizuka M, Nagata H, Takagi K, Iwasaki Y, Shibuya N, Kubota K. Clinical Significance of the C-Reactive Protein to Albumin Ratio for Survival After Surgery for Colorectal Cancer. Ann Surg Oncol. 2016;23:900-7.

11. Mao M, Wei X, Sheng H, Chi P, Liu Y, Huang X, et al. C-reactive protein/albumin and neutrophil/lymphocyte ratios and their combination predict overall survival in patients with gastric cancer. Oncol Lett. 2017;14:7417-24.

12. Liu X, Sun X, Liu J, Kong P, Chen S, Zhan Y, et al. Preoperative C-Reactive Protein/Albumin Ratio Predicts Prognosis of Patients after Curative Resection for Gastric Cancer. Transl Oncol. 2015;8:339-45.

13. Sun F, Ge X, Liu Z, Du S, Ai S, Guan W. Postoperative C-reactive protein/albumin ratio as a novel predictor for short-term complications following gastrectomy of gastric cancer. World J Surg Oncol. 2017;15. doi:10.1186/s12957-017-1258-5.

14. Edge SB, Compton CC. The American Joint Committee on Cancer: the 7th edition of the AJCC cancer staging manual and the future of TNM. Ann Surg Oncol. 2010;17:1471-4.

15. Hashimoto S, Tominaga T, Nonaka T, Hamada K, Araki M, Takeshita H, et al. The C-reactive protein to albumin ratio predicts postoperative complications in oldest-old patients with colorectal cancer. Int J Colorectal Dis. 2020.

16. Sah BK, Zhu ZG, Chen MM, Yan M, Yin HR, Zhen LY. Gastric cancer surgery and its hazards: post operative infection is the most important complication. Hepatogastroenterology. 2008;55:2259-63.

17. Nozoe T, Matsumata T, Sugimachi K. Preoperative elevation of serum C-reactive protein is related to impaired immunity in patients with colorectal cancer. Am J Clin Oncol. 2000;23:263-6.

18. De Magistris L, Paquette B, Orry D, Facy O, Di Giacomo G, Rat P, et al. Preoperative inflammation increases the risk of infection after elective colorectal surgery: results from a prospective cohort. Int J Colorectal Dis. 2016;31:1611-7.

19. Putwatana P, Reodecha P, Sirapo-ngam Y, Lertsithichai P, Sumboonnanonda K. Nutrition screening tools and the prediction of postoperative infectious and wound complications: comparison of methods in presence of risk adjustment. Nutrition. 2005;21:691-7.

20. Rizzo A, Cengel KA. Anti-inflammatory therapy for pancreatic cancer: a sorely needed advance in therapeutics. Cancer Biol Ther. 2008;7:1051-2.

21. Langman MJ, Cheng KK, Gilman EA, Lancashire RJ. Effect of anti-inflammatory drugs on overall risk of common cancer: case-control study in general practice research database. BMJ. 2000;320:1642-6. 